\title{
Boundary Layer Stagnation-Point Slip Flow and Heat Transfer towards a Shrinking/Stretching Cylinder over a Permeable Surface
}

\author{
Nor Azian Aini Mat' ${ }^{1}$, Norihan Md. Arifin ${ }^{2 *}$, Roslinda Nazar ${ }^{3}$, Norfifah Bachok ${ }^{4}$ \\ ${ }^{1}$ Faculty of Science and Mathematics, Universiti Pendidikan Sultan Idris, Perak, Malaysia \\ ${ }^{2}$ Institute for Mathematical Research, Universiti Putra Malaysia, Selangor, Malaysia \\ ${ }^{3}$ School of Mathematical Sciences, Universiti Kebangsaan Malaysia, Selangor, Malaysia \\ ${ }^{4}$ Department of Mathematics, Faculty of Science, Universiti Putra Malaysia, Selangor, Malaysia \\ Email: ${ }^{*}$ norihanarifin@yahoo.com
}

Received 14 February 2015; accepted 4 March 2015; published 10 March 2015

Copyright (C) 2015 by authors and Scientific Research Publishing Inc.

This work is licensed under the Creative Commons Attribution International License (CC BY).

http://creativecommons.org/licenses/by/4.0/

(c) (i) Open Access

\section{Abstract}

In this paper, the boundary layer stagnation-point slip flow and heat transfer towards a shrinking/stretching cylinder over a permeable surface is considered. The governing equations are first transformed into a system of non-dimensional equations via the non-dimensional variables, and then into self-similar ordinary differential equations before they are solved numerically using the shooting method. Numerical results are obtained for the skin friction coefficient and the local Nusselt number as well as the velocity and temperature profiles for some values of the governing parameters, namely the velocity slip parameter $(\delta)$, the thermal slip parameter $(\beta)$, the curvature parameter $(\gamma)$ and the velocity ratio parameter $(c / a)$. The physical quantities of interest are the skin friction coefficient and the local Nusselt number measured by $f^{\prime \prime}(0)$ and $-\theta^{\prime}(0)$, respectively. The numerical results show that the velocity slip parameter $\delta$ increases the heat transfer rate at the surface, while the thermal slip parameter $\beta$ decreases it. On the other hand, increasing the velocity slip parameter $\delta$ causes the decrease in the flow velocity. Further, it is found that the solutions for a shrinking cylinder $(c / a<0)$ are non-unique with dual solutions, which is different from a stretching cylinder $(c / a>0)$ case. Finally, it is also found that the values of $f^{\prime \prime}(0)$ and $-\theta^{\prime}(0)$ increase as the curvature parameter $\gamma$ increases.

\footnotetext{
*Corresponding author.
}

How to cite this paper: Mat, N.A.A., Arifin, N.Md., Nazar, R. and Bachok, N. (2015) Boundary Layer Stagnation-Point Slip Flow and Heat Transfer towards a Shrinking/Stretching Cylinder over a Permeable Surface. Applied Mathematics, 6, 466475. http://dx.doi.org/10.4236/am.2015.63044 


\section{Keywords}

\section{Boundary Layer, Heat Transfer, Numerical Solution, Shrinking/Stretching Cylinder, Slip Flow, Stagnation-Point, Suction/Injection}

\section{Introduction}

The problem of flow and heat transfer induced by continuous shrinking or stretching surfaces has received considerable attention in recent years because it is an important type of flow occurring in many engineering disciplines. The stagnation point flow problems have been extensively studied by several authors. The problem of flow due to a shrinking or stretching sheet has been later extended to many flow situations. Crane [1] was the first studied the steady boundary layer flow of a viscous fluid due to a linearly stretching surface. Later, Chiam [2] extended the works of Crane [1] to stagnation-point flow over a stretching sheet. Wang [3] first investigated the stagnation-point flow towards a shrinking sheet for both two-dimensional and axiymmetric cases. He found that problem have the dual solutions as well as unique solution for a specific values of the ratio of shrinking.

The problem of unsteady stagnation-point flow of a viscous and incompressible fluid by considering both the stretching and shrinking sheet situations have been investigated by Fan et al. [4]. On the other hand, Bachok et $a l$. [5] discussed the effect of melting on boundary layer stagnation-point flow towards a stretching or shrinking sheet. Ahmad et al. [6] investigated the behaviour of the steady boundary layer flow and heat transfer of a micropolar fluid near the stagnation point on a stretching vertical surface with prescribed skin friction. Lok et al. [7] studied the steady axisymmetric stagnation point flow of a viscous and incompressible fluid over a shrinking circular cylinder with mass transfer (suction). Bhattacharyya et al. [8] analyzed the effects of partial slip on the steady boundary layer stagnation-point flow of an incompressible fluid and heat transfer towards a shrinking sheet. This investigation explores the conditions of the non-existence, existence, uniqueness and duality of the solutions of self-similar equations numerically. They also studied the same case but under the condition of unsteady-state towards a stretching. Stagnation-point flow and heat transfer over an exponentially shrinking sheet was analyzed by Bhattacharyya and Vajravelu [9]. They obtained dual solutions for the velocity and the temperature fields and also they observed that their boundary layers are thinner for the first solution.

Therefore, the present investigation deals with the effects of partial slip on the boundary layer stagnationpoint flow and heat transfer towards a shrinking/stretching cylinder over a permeable surface.

\section{Problem Formulation}

Consider the steady stagnation-point flow towards a horizontal linearly stretching/shrinking cylinder with radius $R$ placed in an incompressible viscous fluid of constant temperature $T_{w}$. Using boundary layer approximation, the equations of motion and temperature distribution may be written in usual notations as below

$$
\begin{gathered}
\frac{\partial}{\partial x}(r u)+\frac{\partial}{\partial r}(r v)=0 \\
u \frac{\partial u}{\partial x}+v \frac{\partial u}{\partial r}=U \frac{\mathrm{d} U}{\mathrm{~d} x}+v\left(\frac{\partial^{2} u}{\partial r^{2}}+\frac{1}{r} \frac{\partial u}{\partial r}\right) \\
u \frac{\partial T}{\partial x}+v \frac{\partial T}{\partial r}=\alpha\left(\frac{\partial^{2} T}{\partial r^{2}}+\frac{1}{r} \frac{\partial T}{\partial r}\right)
\end{gathered}
$$

where $x$ and $r$ are coordinates measured along the surface of the cylinder and in the radial direction, respectively, with $u$ and $v$ being the corresponding velocity components and $U(x)=a x$ is the straining velocity of the stagnation-point flow. Further, $T$ is the temperature in the boundary layer, $v$ is the kinematic viscosity coefficient and $\alpha$ is the thermal diffusivity.

The appropriate boundary conditions for the velocity components with partial slip condition at the wall and for the temperature are given by 


$$
\begin{gathered}
u=c x+L(\partial u / \partial r), \quad v=v_{w}(x), \\
T=T_{w}+D(\partial T / \partial r) \quad \text { at } r=R . \\
u \rightarrow U(x)=a x, \quad T \rightarrow T_{\infty} \quad \text { as } r \rightarrow \infty
\end{gathered}
$$

where $c$ and $a(>0)$ are the shrinking/stretching rate (of the surface) and the straining rate parameters, respectively, with $c<0$ for a shrinking cylinder, $c>0$ for a stretching cylinder and $L$ is the velocity slip factor, $D$ is the thermal slip factor, $v=v_{w}(x)$ is the mass transfer velocity with $v_{w}(x)>0$ for suction and $v_{w}(x)<0$ for injection $T_{w}$ is the surface temperature and $T_{\infty}$ is the free stream temperature, both are assumed to be constant with $T_{w}>T_{\infty}$.

Next, we introduce the transformation as follows:

$$
\psi=(a v)^{1 / 2} x R f(\eta), \quad T=T_{\infty}+\left(T_{w}-T_{\infty}\right) \theta(\eta)
$$

where $\eta$ is the similarity variable and is defined as $\eta=\frac{r^{2}-R^{2}}{2 R}(a / v)^{1 / 2}$ and $\psi$ is the stream function define as $u=r^{-1} \partial \psi / \partial r$ and $v=-r^{-1} \partial \psi / \partial x$, which identically satisfies Equation (1). Since this problem have effects of suction and injection, we have consider that $v_{w}(x)$ in Equation (4) has the following expression,

$$
v_{w}(x)=-\frac{1}{r}\left(\frac{v U}{x}\right)^{1 / 2} R f_{w}
$$

where $f_{w}=f(0)$ is a non-dimensional constant determines the transpiration rate, with $f_{w}>0$ is the constant suction parameter, $f_{w}<0$ is the constant injection parameter and $f_{w}=0$ for an impermeable surface. Physically permeable surface (suction/injection) allows fluid to pass through either in or out. Using (7) we obtain the following self-similar equations

$$
\begin{gathered}
(1+2 \gamma \eta) f^{\prime \prime \prime}+2 \gamma f^{\prime \prime}+f f^{\prime \prime}+1-f^{\prime 2}=0, \\
(1+2 \gamma \eta) \theta^{\prime \prime}+2 \gamma \theta^{\prime}+\operatorname{Pr} f \theta^{\prime}=0
\end{gathered}
$$

subject to the boundary conditions (5) and (6) which become

$$
\begin{aligned}
& f(0)=f_{W}, \quad f^{\prime}(0)=c / a+\delta f^{\prime \prime}(0), \\
& \theta(0)=1+\beta \theta^{\prime}(0), \\
& f^{\prime}(\infty) \rightarrow 1, \quad \theta(\infty) \rightarrow 0,
\end{aligned}
$$

where $\gamma$ is the curvature parameter and $\operatorname{Pr}$ is the Prandtl number defined respectively as

$$
\gamma=\left(\frac{v}{a R^{2}}\right)^{1 / 2}, \quad \operatorname{Pr}=\frac{v}{\alpha}
$$

and $c / a$ is the velocity ratio parameter, $\delta=L \frac{r}{R}(a / v)^{1 / 2}$ is the velocity slip parameter and $\beta=D \frac{r}{R}(a / v)^{1 / 2}$ is the thermal slip parameter.

The main physical quantities of interest are the value of $f^{\prime \prime}(0)$, being a measure of the skin friction coefficient (shear stress at the surface), and $-\theta^{\prime}(0)$, the local Nusselt number (rate of heat transfer at the surface). Our main aim is to find how the values of $f^{\prime \prime}(0)$ and $-\theta^{\prime}(0)$ vary in terms of the parameters $f_{w}, \beta, \delta$, $\delta$ and $c / a$. The present problem reduces to those considered by Bhattacharyya et al. [8] when $\gamma=0$ (flat plate), $\delta \neq 0$ and $\beta=0$.

\section{Results and Discussion}

Equations (8) and (9) with the boundary conditions (10) are solved numerically using the shooting method. Table 1 shows the variations of $c / a_{\text {crit }}$ (critical values of $c / a$ ) with parameters $\delta$ and $\gamma$ when $f_{w}=0$, $\beta=0$ (without thermal slip) and $\operatorname{Pr}=1$. In order to verify the accuracy of the present method, we have com- 


\begin{tabular}{cccc} 
Table 1. Variations of $c / a_{\text {crit }}$ & with parameters $\delta$ and $\gamma$ when $f_{w}=0, \beta=0$ and $\operatorname{Pr}=1$. \\
\hline$\delta$ & $\gamma$ & Bhattacharyya et al. [8] & Present \\
\hline 0 & 0 & -1.2465 & -1.24657 \\
& 0.2 & & -1.38090 \\
0.4 & -1.31067 & -1.49353 \\
0.1 & 0 & & -1.31067 \\
& 0.2 & & -1.45598 \\
& 0.4 & & -1.58171
\end{tabular}

pared the present results obtained with those of Bhattacharyya et al. [8] for $\gamma=0$ (flat plate), $\delta \neq 0$. This comparison is given in Table 1 and we can see that there is a very good agreement between these results, and thus we are confident that the numerical results obtained are accurate. Table 2 presents the numerical values of $f^{\prime \prime}(0)$ with respect to $\delta, c / a$ and $\gamma$ when $f_{w}=0, \beta=0.2$ (with thermal slip) and $\operatorname{Pr}=1$. From this table it is observed that with increasing $\gamma$, the values of $f^{\prime \prime}(0)$ increase for several values of $\delta$. Figure 1 and Figure 2 show the variations of $f^{\prime \prime}(0)$ and $-\theta^{\prime}(0)$ with $c / a$, respectively, for various values of $\gamma$ when $f_{w}=0, \delta=0.1, \beta=0.2$ and $\operatorname{Pr}=1$. As the curvature parameter $\gamma$ increases, the skin friction coefficient $f^{\prime \prime}(0)$ and the local Nusselt number $-\theta^{\prime}(0)$ increase. On the other hand, $-\theta^{\prime}(0)$ increase with the increasing $c / a$. It is show that for increasing slip at the sheet, the vorticity generated due to the shrinking. velocity is slightly reduced and hence with the same straining, vorticity remains confined within the boundary layer for larger shrinking velocity also and consequently the steady solution is also possible for some large value of $c / a$. Figure 3 and Figure 4 represent the variations of $f^{\prime \prime}(0)$ and $-\theta^{\prime}(0)$ for various values of $\delta$ when $f_{w}=0, \gamma=0.2, \beta=0.2$ and $\operatorname{Pr}=1$. It is shown that the velocity slip parameter $\delta$ decreases the shear stress rate but increases the heat transfer rate at the surface. It is seen that the region of non-unique solutions exist for $c / a_{\text {crit }} \leq c / a \leq-1$ and the solution is unique for $c / a>-1$. The value of $f^{\prime \prime}(0)$ is zero when $c / a=1$ for all values of $\gamma$ and $\delta$ considered and $c / a=0$ is the Hiemenz flow while $c / a=1$ is a degenerate inviscid flow where the stretching cylinder matches the conditions at infinity.

Figure 5 shows the variations of $-\theta^{\prime}(0)$ for various values of $\beta$ (thermal slip parameter) for $f_{w}=0$, $\delta=0.1, \gamma=0.2$ and $\operatorname{Pr}=1$. As $c / a$ increases, the heat transfer rate measured by $-\theta^{\prime}(0)$ increases too, while it decreases with the thermal slip parameter, $\beta$. It should be pointed out that the effect of thermal slip parameter $\beta$ on the shear stress is not significant so it is not presented here. Figure 6 and Figure 7 show the variations of $f^{\prime \prime}(0)$ and $-\theta^{\prime}(0)$ with $\delta$, respectively, for various values of $\gamma$ when $f_{w}=0, \beta=0.2$, $c / a=-1.2$ and $\operatorname{Pr}=1$. The values of $f^{\prime \prime}(0)$ and $-\theta^{\prime}(0)$ increase with $\gamma$ for the first solution and for the second solution the effect is completely the opposite. Further, as $\delta$ increases, $-\theta^{\prime}(0)$ increases too. Figure 8 shows the variations of $-\theta^{\prime}(0)$ with $\beta$ for various values of $\gamma$ when $f_{w}=0, \delta=0.1, c / a=-1.2$ and $\operatorname{Pr}=1$. The values of $-\theta^{\prime}(0)$ increase with $\gamma$ for the first and the second solutions. It is also noted that $-\theta^{\prime}(0)$ is decreasing as $\beta$ increases.

Figure 9 and Figure 10 represent the variations of $f^{\prime \prime}(0)$ and $-\theta^{\prime}(0)$ with $f_{w}$ for different values of $\delta$ when $\beta=0.2, \gamma=0.2, c / a=-1.2$ and $\operatorname{Pr}=1$. It is found that the values of $f^{\prime \prime}(0)$ and $-\theta^{\prime}(0)$ increase with $\delta$ for the first solution and for the second solution the effect is completely the opposite. The behavior are similar as $f_{w}$ increases. Figure 11 and Figure 12 show the velocity profiles $f^{\prime}(\eta)$ and the temperature profiles $\theta(\eta)$ for various values of $\gamma$ when $f_{w}=0, c / a=-1.2, \operatorname{Pr}=1, \delta=0.1$ and $\beta=0.2$. Figure 13 and Figure 14 represent the velocity profiles $f^{\prime}(\eta)$ and temperature profiles $\theta(\eta)$ for various values of $f_{w}$ when $c / a=-1.2, \operatorname{Pr}=1, \delta=0.1, \gamma=0.2$ and $\beta=0.2$. It is shown that all the curves approach the far field boundary conditions asymptotically. Further, these figures also support the existence of dual nature of the solutions as presented in the earlier figures where the boundary layer thickness for the first solution is smaller than that of the second solution. 


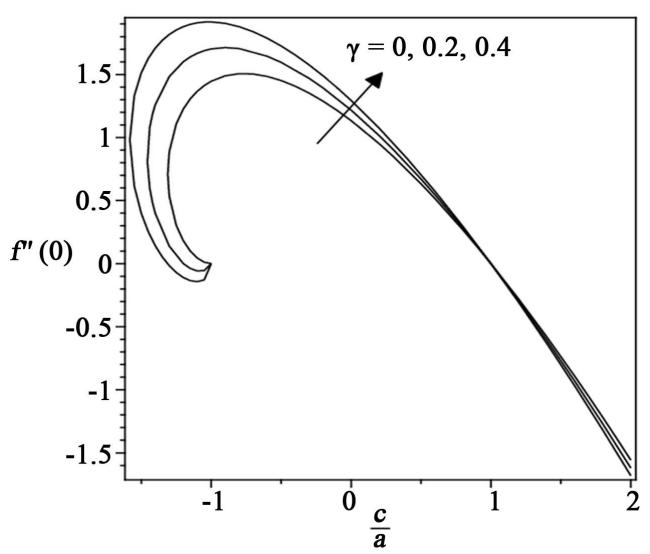

Figure 1. Variations of $f^{\prime \prime}(0)$ with $c / a$ for various values of $\gamma$ when $f_{w}=0, \delta=0.1$, $\beta=0.2$ and $\operatorname{Pr}=1$.

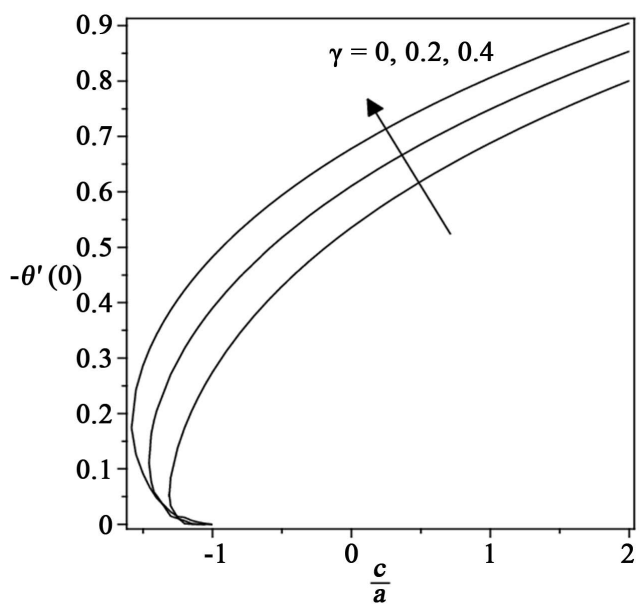

Figure 2. Variations of $-\theta^{\prime}(0)$ with $c / a$ for various values of $\gamma$ when $f_{w}=0, \delta=0.1$, $\beta=0.2$ and $\operatorname{Pr}=1$.

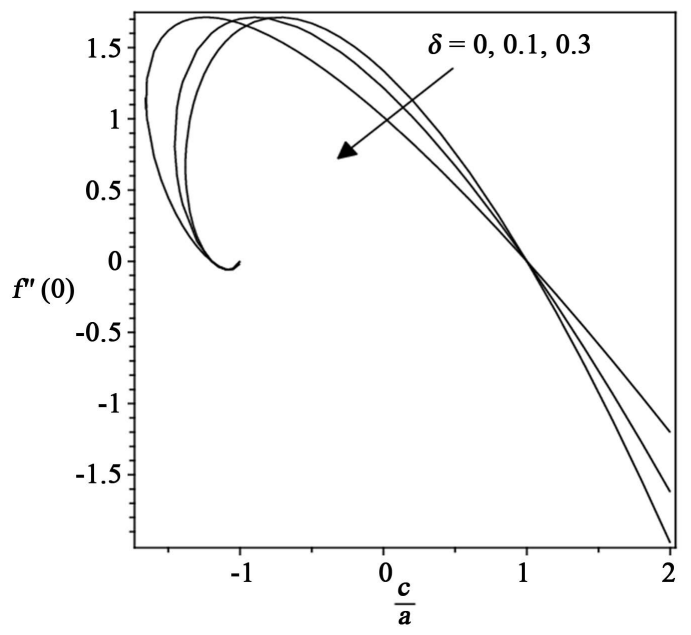

Figure 3. Variations of $f^{\prime \prime}(0)$ with $c / a$ for various values of $\delta$ when $f_{w}=0, \gamma=0.2$, $\beta=0.2$ and $\operatorname{Pr}=1$. 


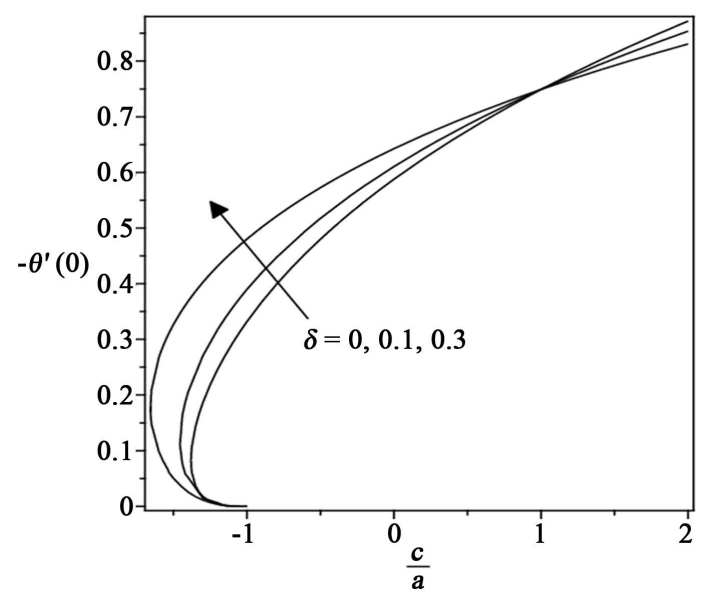

Figure 4. Variations of $-\theta^{\prime}(0)$ with $c / a$ for various values of $\delta$ when $f_{w}=0, \gamma=0.2$, $\beta=0.2$ and $\operatorname{Pr}=1$.

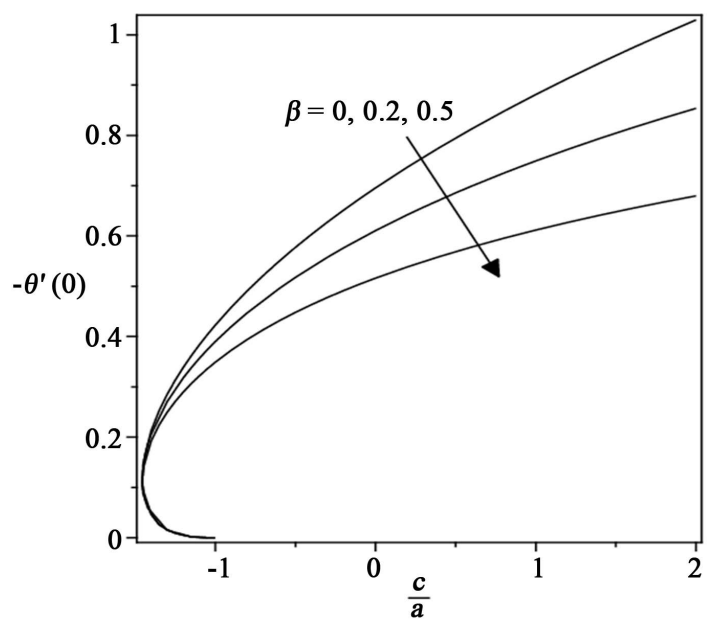

Figure 5. Variations of $-\theta^{\prime}(0)$ with $c / a$ for various values of $\beta$ when $f_{w}=0, \delta=0.1$, $\gamma=0.2$ and $\operatorname{Pr}=1$.

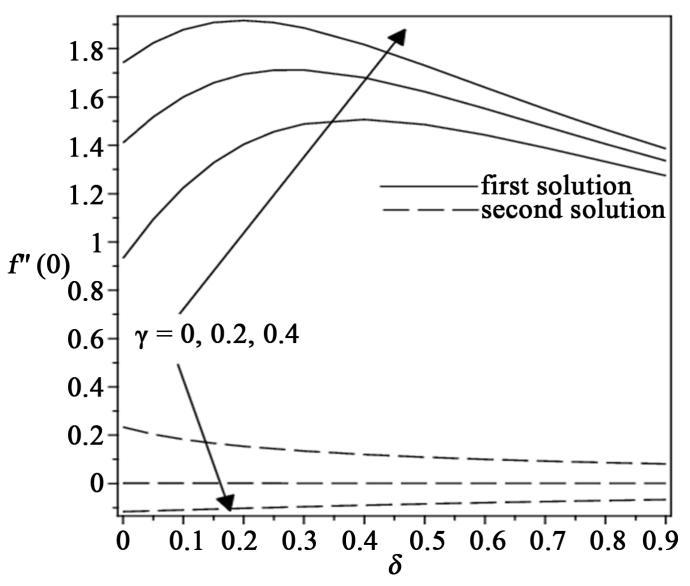

Figure 6. Variations of $f^{\prime \prime}(0)$ with $\delta$ for various values of $\gamma$ when $f_{w}=0, \beta=0.2$, $c / a=-1.2$ and $\operatorname{Pr}=1$. 


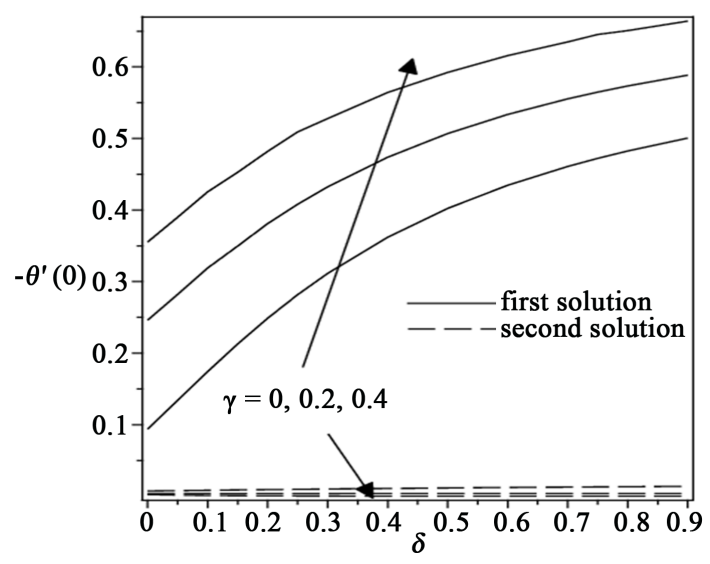

Figure 7. Variations of $-\theta^{\prime}(0)$ with $\delta$ for various values of $\gamma$ when $f_{w}=0, \beta=0.2$, $c / a=-1.2$ and $\operatorname{Pr}=1$.

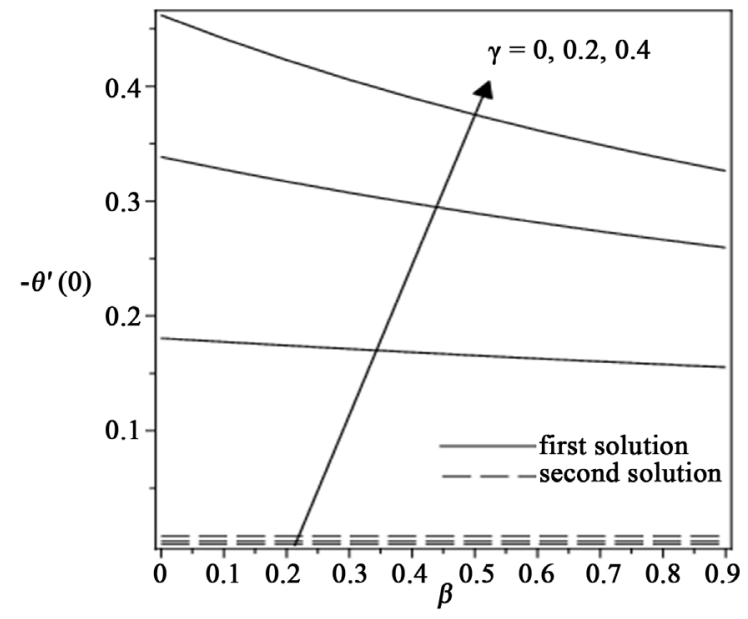

Figure 8. Variations of $-\theta^{\prime}(0)$ with $\beta$ for various values of $\gamma$ when $f_{w}=0, \delta=0.1$, $c / a=-1.2$ and $\operatorname{Pr}=1$.

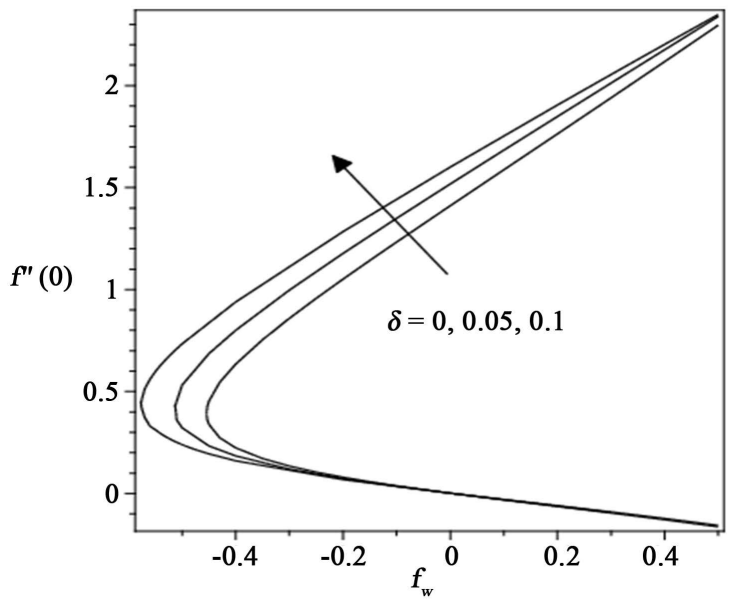

Figure 9. Variations of $f^{\prime \prime}(0)$ with $f_{w}$ for different values of $\delta$ when $\beta=0.2$, $\gamma=0.2, c / a=-1.2$ and $\operatorname{Pr}=1$. 


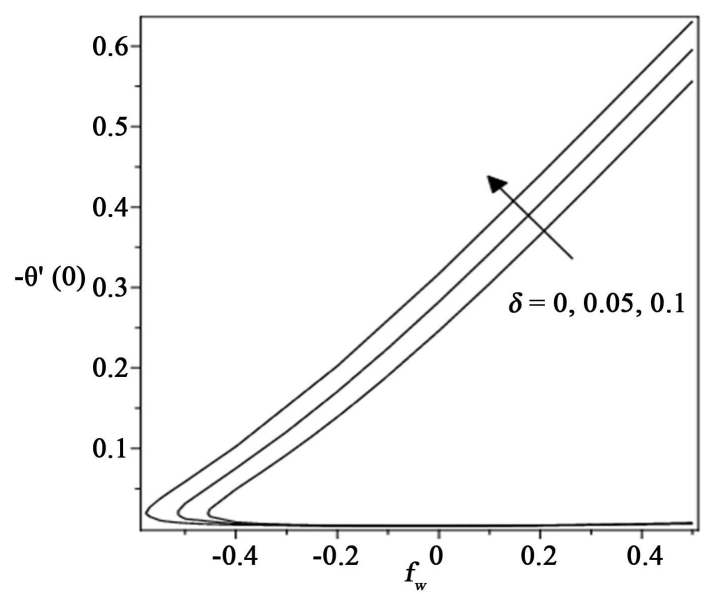

Figure 10. Variations of $-\theta^{\prime}(0)$ with $f_{w}$ for different values of $\delta$ when $\beta=0.2$, $\gamma=0.2, c / a=-1.2$ and $\operatorname{Pr}=1$.

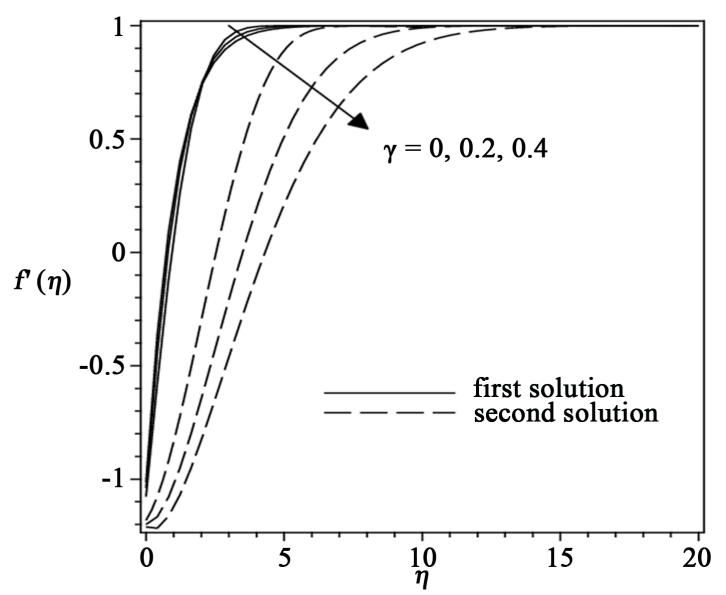

Figure 11. Velocity profiles $f^{\prime}(\eta)$ for various values of $\gamma$ when $f_{w}=0, c / a=-1.2$, $\operatorname{Pr}=1, \quad \delta=0.1$ and $\beta=0.2$.

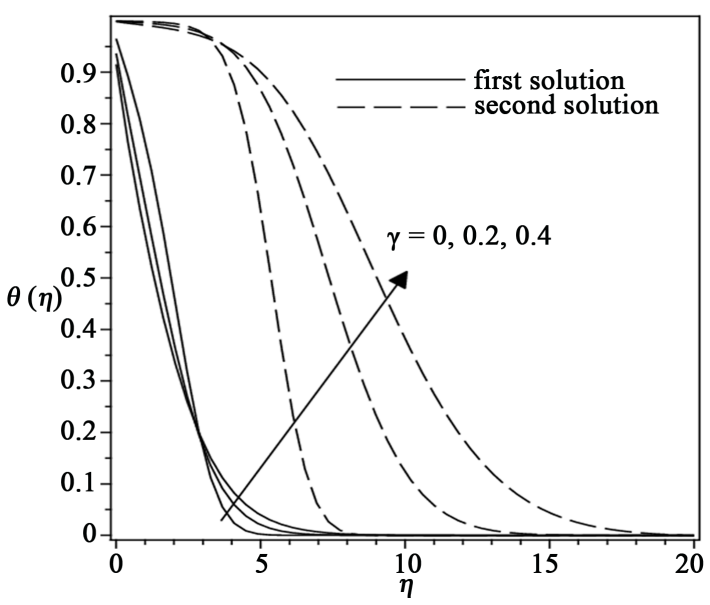

Figure 12. Temperature profiles $\theta(\eta)$ for various values of $\gamma$ when $f_{w}=0, c / a=-1.2$, $\operatorname{Pr}=1, \delta=0.1$ and $\beta=0.2$. 


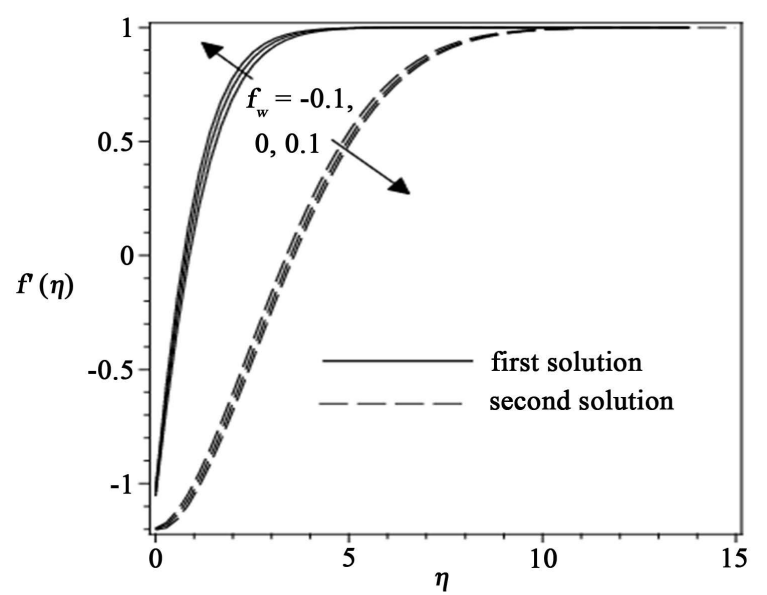

Figure 13. Velocity profiles $f^{\prime}(\eta)$ for various values of $f_{w}$ when $c / a=-1.2, \operatorname{Pr}=1$, $\delta=0.1, \gamma=0.2$ and $\beta=0.2$.

Table 2. Variations of $f^{\prime \prime}(0)$ with respect to $\delta, c / a$ and $\gamma$ when $f_{w}=0, \beta=0.2$ and $\operatorname{Pr}=1$. ( ) is the second solution.

\begin{tabular}{|c|c|c|c|c|}
\hline$\delta$ & $c / a$ & $\gamma=0$ & $\gamma=0.2$ & $\gamma=0.4$ \\
\hline \multirow[t]{8}{*}{0} & -1.2 & 0.932474 & 1.410611 & 1.742367 \\
\hline & & $(0.233650)$ & $(0.001521)$ & $(-0.116572)$ \\
\hline & -1.15 & 1.082232 & 1.485004 & 1.791168 \\
\hline & & $(0.116702)$ & $(-0.040137)$ & $(-0.138295)$ \\
\hline & -0.5 & 1.495670 & 1.670570 & 1.830761 \\
\hline & 0 & 1.232588 & 1.337874 & 1.436692 \\
\hline & 0.5 & 0.713295 & 0.762907 & 0.810058 \\
\hline & 2 & -1.887307 & -1.977842 & -2.065444 \\
\hline \multirow[t]{8}{*}{0.1} & -1.2 & 1.224941 & 1.600250 & 1.878646 \\
\hline & & $(0.182621)$ & $(0.001374)$ & $(-0.109447)$ \\
\hline & -1.15 & 1.306265 & 1.638745 & 1.897256 \\
\hline & & $(0.100177)$ & $(-0.037795)$ & $(-0.134217)$ \\
\hline & -0.5 & 1.451720 & 1.595891 & 1.725654 \\
\hline & 0 & 1.134281 & 1.217492 & 1.294264 \\
\hline & 0.5 & 0.632869 & 0.670662 & 0.705994 \\
\hline & 2 & -1.557650 & -1.620898 & -1.681197 \\
\hline \multirow[t]{8}{*}{0.2} & -1.2 & 1.404016 & 1.694527 & 1.916706 \\
\hline & & $(0.153829)$ & $(0.001253)$ & $(-0.102528)$ \\
\hline & -1.15 & 1.442545 & 1.706290 & 1.913929 \\
\hline & & $(0.088601)$ & $(-0.035615)$ & $(-0.129281)$ \\
\hline & -0.5 & 1.387061 & 1.503930 & 1.607658 \\
\hline & 0 & 1.042585 & 1.108734 & 1.168950 \\
\hline & 0.5 & 0.567044 & 0.596541 & 0.623772 \\
\hline & 2 & -1.331322 & -1.378600 & -1.423186 \\
\hline
\end{tabular}




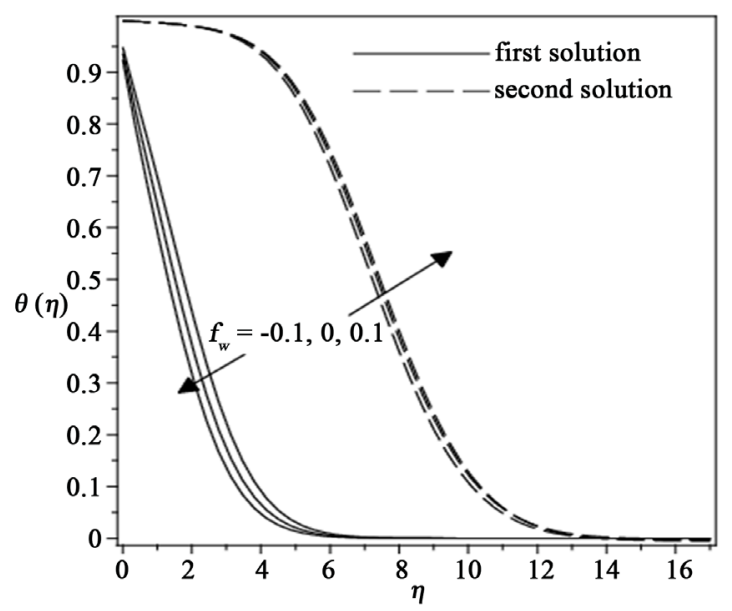

Figure 14. Temperature profiles $\theta(\eta)$ for various values of $f_{w}$ when $c / a=-1.2, \operatorname{Pr}=1$, $\delta=0.1, \gamma=0.2$ and $\beta=0.2$.

\section{Conclusion}

The study of boundary layer stagnation-point slip flow and heat transfer towards a shrinking/stretching cylinder over a permeable surface has been investigated numerically using the shooting method. Comparisons of the present results with those of Bhattacharyya et al. [8] are in excellent agreement. The velocity slip parameter $(\delta)$ increases the heat transfer rate at the surface, while the thermal slip parameter $(\beta)$ decreases it. On the other hand, it is found that dual solutions exist for the shrinking cylinder when $c / a<0$. Finally, as the curvature parameter $(\gamma)$ increases, the values of $f^{\prime \prime}(0)$ and $-\theta^{\prime}(0)$ increase too, similar with the effect of suction/injection parameter $\left(f_{w}\right)$ for the first solution.

\section{Acknowledgements}

The authors gratefully acknowledged the financial support received in the form of a FRGS research grant from the Ministry of Education, Malaysia.

\section{References}

[1] Crane, L.J. (1970) Flow Past a Stretching Plate. ZAMP, 21, 645-647. http://dx.doi.org/10.1007/BF01587695

[2] Chiam, T.C. (1994) Stagnation-Point Flow towards a Stretching Plate. Journal of Physical Society of Japan, 63, 24432444. http://dx.doi.org/10.1143/JPSJ.63.2443

[3] Wang, C.Y. (2008) Stagnation Flow towards a Shrinking Sheet. International Journal of Non-Linear Mechanics, 43, 377-382. http://dx.doi.org/10.1016/j.ijnonlinmec.2007.12.021

[4] Fan, T., Xu, H. and Pop, I. (2010) Unsteady Stagnation Flow and Heat Transfer towards a Shrinking Sheet. International Communications in Heat and Mass Transfer, 37, 1440-1446. http://dx.doi.org/10.1016/j.icheatmasstransfer.2010.08.002

[5] Bachok, N., Ishak, A. and Pop, I. (2010) Melting Heat Transfer in Boundary Layer Stagnation-Point Flow towards a Stretching/Shrinking Sheet. Physics Letters A, 374, 4075-4079. http://dx.doi.org/10.1016/j.physleta.2010.08.032

[6] Ahmad, K., Nazar, R. and Pop, I. (2011) Boundary Layer Flow and Heat Transfer of a Micropolar Fluid near The Stagnation Point on a Stretching Vertical Surface with Presribed Skin Friction. International Journal of Minerals, Metallurgy and Materials, 18, 502-507. http://dx.doi.org/10.1007/s12613-011-0469-y

[7] Lok, Y.Y., Ishak, A. and Pop, I. (2011) MHD Stagnation Point Flow with Suction towards a Shrinking Sheet. Sains Malaysiana, 40, 1179-1186.

[8] Bhattacharyya, K., Mukhopadhyay, S. and Layek, G.C. (2011) Slip Effects on Boundary Layer Stagnation-Point Flow and Heat Transfer towards a Shrinking Sheet. International Journal of Heat and Mass Transfer, 54, 308-331. http://dx.doi.org/10.1016/j.ijheatmasstransfer.2010.09.041

[9] Bhattacharyya, K. and Vajravelu, K. (2012) Stagnation-Point Flow and Heat Transfer over an Exponentially Shrinking Sheet. Communications in Nonlinear Science and Numerical Simulation, 17, 2728-2734. 